\title{
RETOS Y PERSPECTIVAS DE LA UNIVERSIDAD EN UN CONTEXTO DE CAMBIO
}

\author{
José Fabio Hernández Díaz*
}

El autor hace un análisis del nuevo contexto internacional en que vivimos, considerando elementos sociales, educativos, económicos y cuales son los desafios a los que la educación superior se enfrenta como consecuencia de dichos elementos.

Se profundiza en los análisis internos que la universidad debe llevar a cabo para poder cumplir con las demandas que la sociedad actual le exige.

Finalmente se hacen una serie de recomendaciones en aspectos como gestión universitaria, financiamiento, acceso a la educación superior, la docencia en la universidad, la investigación universitaria y la vinculación con el sector externo.
This article analizes the new international context in which we are living, considering social, educational and economic factors and the main challenges that higher education confronts as a result of those factors. It emphasizes the internal analysis that universities must undertake in order to satisfy the demands of today's society:

It also presents a series of recommendations on several aspects such as university management, university financing, college opportunities, college teaching, university research, and the relationships between universities and societn:

* Master en Educación. con énfasis en Docencia Universitaria y Licenciado en la Enseñanza de la Matemática de la Universidad Nacional. Actualmente es Coordinador del Centro de Desarrollo Académico del Instituto Tecnológico de Costa Rica., ha sido Profesor en la Escuela de Matemática del Instituto Tecnológico de Costa Rica., desde 1985. También es Profesor de la División de Educología del Centro de Investigación en Docencia y Educación de la Universidad Nacional, desde 1992. 


\section{Introducción}

La profundidad y velocidad de los cambios que experimenta la sociedad mundial en prácticamente todos los órdenes han colocado a las instituciones de educación superior ante la necesidad de revisar sus planteamientos y renovar sus acciones y nexos con la sociedad.

Las profundas transformaciones de que somos testigos nos señalan que la sociedad actual demanda una acción protagónica de las instituciones de educación superior. Los acelerados progresos en la tecnología propician a su vez un cambio en los procesos de generación y transmisión del conocimiento. Por otro lado el contexto internacional es altamente competitivo y demanda por lo tanto en forma creciente recursos humanos muy calificados. Estos hechos, entre otros, plantean a las universidades nuevas interrogantes en la búsqueda de la excelencia y su pertinencia histórica y social.

La Educación Superior debe estar en capacidad de construir alternativas innovadoras y de alta calidad de modo que posibilite a los países, particularmente de nuestra región, responder a los nuevos retos sociales, económicos, culturales y ecológicos, fortaleciendo al mismo tiempo los procesos democráticos y promoviendo un desarrollo con equidad. Todas estas demandas que plantea la sociedad deben traducirse en procesos de modernización y transformación de las instituciones de educación superior.

\section{Aspectos contextuales}

Hasta hace pocos años, existían en el mundo dos superpotencias, la Unión Soviética y los Estados Unidos. Al caer el bloque socialista se consolidó un solo polo, pero casi al mismo tiempo, dos nuevos y poderosos centros económicos se están consolidando; uno en Europa y otro en el Oriente asiático.

La Comunidad Económica Europea surge como una poderosa conjugación de fuerzas económicas, políticas, culturales y científicas que le permitirán asumir un destacado papel en el escenario político y económico mundial. El «viejo mundo» ha mostrado una capacidad y vitalidad sorprendente al adoptar medidas revolucionarias para establecer un mercado único, derrumbando las fronteras, facilitando la libre circulación de los ciudadanos, el comercio, la empresa, las inversiones y la moneda.

En los campos científico y tecnológico, los europeos tienen bases sólidas en las universidades que a lo largo de las últimas décadas han cambiado su aislamiento individualista por la colaboración y la realización de proyectos de investigación de gran envergadura y en estrecha colaboración interinstitucional. 
También el bloque asiático se ha posicionado en el panorama mundial. y hoy día países como Japón. China. Malasia y otros han obtenido un desarrollo acelerado a nivel mundial y compiten abiertamente con Estados Unidos y Europa.

En esta nueva configuración sociopolítica, los países de América Latina se encuentran profundamente divididos, pero empeñados en su desarrollo económico y social y en disfrutar de los avances tecnológicos. Para lograrlo se verán obligados a redefinir sus estrategias y alianzas de modo que no se marginen del desarrollo mundial.

Como consecuencia inmediata de este nuevo contexto internacional ha surgido en sustitución de la carrera armamentista una creciente y despiadada competencia económica entre los países ricos Si bien ha disminuido la amenaza de destrucción nuclear para la humanidad, hoy se vive una constante preocupación por la hegemonía económica mediante el dominio del capital y la tecnología.

Tunnerman (1998, p.24) señala que el reciente Informe de la Comisión Internacional sobre la Educación para el Siglo XXI, conocido como Informe Delors, sostiene que «el fenómeno de la globalización es hoy día el más importante, el más dominante y el que, de un modo u otro, más influye en la vida diaria de todas las personas». Luego este mismo autor afirma que la polarización económica a nivel mundial, se incrementa constantemente. Según el Programa de las Naciones Unidas para el Desarrollo (PNUD), casi el 80\% del producto interno bruto mundial corresponde a los países industrializados y solo el $20 \%$ a los países en desarrollo, no obstante que en éstos vive el $80 \%$ de la población mundial.

Otro dato preocupante lo aporta la revista Forbes Magazine: existen 358 personas cuyo capital es de 762 mil millones de dólares, esta cantidad equivale al ingreso per cápita del $45 \%$ de la población mundial. (Tunnerman, 1998, p.26).

En la década de los ochenta, las exportaciones en América Latina crecieron aproximadamente un $60 \%$, mientras que las importaciones disminuyeron en casi un $15 \%$. Sin embargo, las condiciones estructurales que crean la deuda externa, los desiguales términos de intercambio y la fuga de capitales provocaron que América Latina transfiriera a América del Norte, descapitalizándose, una cantidad aproximada de 500 mil millones de dólares. Tunnerman (1998, p.27).

El proceso de globalización obliga a los diferentes Estados a implementar nuevos enfoques y políticas que fomenten la negociación y la competitividad, procurando su inserción en la economía internacional. Las instituciones de educación superior tienen que participar de este compromiso, de modo que contribuyan a la formulación de políticas en este sentido. Para desarrollar la competitividad, nuestros países requieren del conocimiento, del desarrollo tecnológico, del acceso y manejo de la información. Esto implica la elevación 
de la calidad de nuestros sistemas educativos y la formación de recursos humanos de alto nivel.

Debe reconocerse también. que si bien el proceso de globalización ha sido un mecanismo de rápida acumulación de capital, especialmente en los países más industrializados, también ha generado importantes disparidades con los países en vías de desarrollo, sobre todo en lo que se refiere al acceso al comercio internacional, al desarrollo científico y tecnológico, a la riqueza mundial y a su adecuada distribución. Como resultado de esta situación se ha ampliado la brecha social y se han empobrecido amplios sectores de la población mundial. Sobre este aspecto, Tunnermann (1998, p.28) al citar al educador brasileño Cristovam Buarque advierte: «necesitamos competitividad económica pero, sobre todo, necesitamos dignidad social. Nada asegura que la primera lleve a la segunda». A la par de la competitividad debemos desarrollar la equidad, la sustentabilidad ambiental y la solidaridad.

En los últimos veinte años muchas empresas extranjeras lograron coordinar sus actividades productivas internacionales, administrar sus filiales en el extranjero y conducir el comercio internacional, gracias al desarrollo y difusión de tecnologías de información, las telecomunicaciones y las innovaciones tecnológicas. Como resultado se ha dado un gran desarrollo de nuevas áreas como biotecnología, nuevos materiales, tecnologías de comunicación y de información, electrónica, el sistema financiero, la industria farmacéutica y otras más.

Este contexto, de rápidos cambios tecnológicos, ofrece grandes retos y nuevas áreas de desarrollo, donde el aporte de la educación y la investigación resultan fundamentales.

\section{Universidad y desarrollo}

Todo este panorama no hace más que confirmar que la educación debe constituirse en la inversión prioritaria de nuestros países, educar para formar recursos humanos del más alto nivel, para fortalecer el progreso científico, tecnológico, para fortalecer la investigación, pero también.educar para compartir.

El sistema educativo debe estar en capacidad de construir alternativas innovadoras y de alta calidad de modo que posibilite a los países, particularmente de nuestra región, responder a los nuevos retos sociales, económicos, culturales y ecológicos, fortaleciendo al mismo tiempo los procesos democráticos y promoviendo un desarrollo con equidad.

La promoción de la educación y la capacitación ha sido un renglón constante en la política de desarrollo económico durante varias décadas. La importancia de la educación con respecto al crecimiento y la educación en el mundo 
desarrollado también ha sido reconocida por mucho tiempo. Bayley (1994, p.461) afirma que «Los historiadores sostienen que la educación pública de las primeras épocas en los Estados Unidos contribuyó a iniciar su era de hegemonía económica. En 1983, la profunda e influyente publicación de Una Nación en Riesgo (Comisión de los EE.UU sobre excelencia en educación), le atribuía los crecientes déficits en el intercambio comercial y los estancados niveles de vida a un sistema educativo en deterioro en ese país. La gente de negocios y los educadores sostenían que el éxito japonés y el alemán en los mercados de exportación se debía a su aparente superioridad en los sistemas educativos.»

La educación y capacitación de los hombres y de las mujeres contribuyen directamente al crecimiento económico. El impacto de la formación se deja ver en los niveles de productividad, en los ingresos que perciben, en la movilidad ocupacional, en la formación en gestión y en la innovación tecnológica.

Puede asegurarse que los trabajadores calificados poseen una «ventaja comparativa» con respecto a la invención y adaptación de nuevas tecnologías. Estos trabajadores difieren de los no calificados en su capacidad para funcionar dentro de este nuevo ambiente, ya que las habilidades realzan la capacidad de enfrentarse con nuevas demandas creadas por la nueva tecnología. Una consecuencia de este hecho es que si la tecnología de un trabajo cambia, la calidad de las habilidades requeridas también debe cambiar.

Para responder adecuadamente ante los nuevos retos, el sistema educativo debe estar en capacidad de dar respuesta a planteamientos como los siguientes: ¿cuáles serán los perfiles de los egresados de las nuevas profesiones?, ¿cuáles son y cómo funcionan las organizaciones que pueden impulsar el nuevo modelo de desarrollo social?, ¿cuáles son los valores y los estilos de vida en que podrán desarrollarse exitosamente las nuevas profesiones?, ¿cómo se desarrollan la creatividad y capacidad de análisis y de síntesis?, ¿cómo pueden los educadores contribuir a desarrollar las características arriba mencionadas?, ¿cómo debe organizarse el proceso de enseñanza y aprendizaje desde el diseño del currículo hasta las actividades que realizan los docentes y los alumnos?.

Existen tendencias en nuestros países que promueven una priorización de los recursos destinados a los primeros niveles educativos en detrimento de la educación superior. En este sentido debe afirmarse que no se pone en duda la importancia de ofrecer a nuestros niños y adolescentes una educación de excelente calidad, pues ésta constituye la base para un aprendizaje y un desarrollo humano permanente sobre el cual podamos construir sistemáticamente nuevos niveles y tipos de educación y capacitación. Sin embargo también se puede afirınar que para proporcionar un sólido ambiente intelectual y científico en la educación básica se requiere el mejoramiento de la enseñanza superior y el desarrollo de la investigación científica. 
Estudios recientes (1994) del Dr. Fernando Reimers, profesor del Harvard Institute for International Development, demuestran que América Latina invierte menos por cada estudiante de nivel superior que las otras regiones del mundo. Esto contradice claramente el criterio de quienes plantean que en nuestra región se invierten más recursos de lo debido en la educación universitaria, en detrimento de la educación primaria y secundaria. Lo que corresponde, entonces, no es redistribuir los escasos recursos existentes entre los distintos niveles educativos, sino transferir más recursos al sector educativo.

Por supuesto, que también nuestros países deben asumir el compromiso de mejorar la calidad, relevancia y pertinencia de todo el sistema educativo, desde una concepción más integral y no segmentada del mismo. Se debe actuar sobre los diversos componentes de dicho sistema: el currículo, los métodos de enseñanza, los recursos disponibles, la formación de los docentes, el ambiente pedagógico, la investigación educativa.

En América Latina, las universidades tienen la mayor responsabilidad en el desarrollo científico-tecnológico, pues en ellas se concentra la mayor parte de la actividad científica. Ellas tienen la gran responsabilidad de contribuir a introducirnos, en forma autónoma y no como un apéndice intelectual, en la civilización científica contemporánea. (Tunnerman, 1998, p.30).

Los países del sudeste asiático, han alcanzado altos niveles de desarrollo en un período relativamente corto. Una de las estrategias seguidas por estos países fue, precisamente, destinar importantes inversiones al sector educativo, en todos sus niveles y en particular en la educación superior. Tunnerman (1998, p.31)

La educación superior se enfrenta en todas partes a desafíos y dificultades relativos a la financiación, la igualdad de condiciones de acceso a los estudios, una mejor capacitación del personal, la mejora y conservación de la calidad de la enseñanza, la investigación y los servicios, la pertinencia de los programas, el establecimiento de acuerdos de cooperación eficaces y la igualdad de acceso a los beneficios que reporta la cooperación internacional.

La segunda mitad del siglo XX, ha sido la época de mayor expansión en la población universitaria a nivel mundial. Según Jofré (1998, p.68), en América del Norte en los años cincuenta se cuadruplicó la tasa de matrícula universitaria y en los años siguientes el crecimiento siguió siendo importante, mientras que en América Latina, el número de estudiantes matriculados se triplicó entre 1970 y 1980. Lamentablemente, también es la época en que se ha agudizado aún más la disparidad, que ya era enorme, entre los países desarrollados, los países en desarrollo y en particular los países pobres, en lo que respecta al acceso a la educación superior y la investigación y los recursos de que disponen. Ha sido igualmente una época de mayor estratificación socioeconómica y de aumento 
de las diferencias de oportunidades de enseñanza dentro de los propios países. incluso en algunos de los más desarrollados y ricos.

Si se carece de instituciones de educación superior e investigación que formen a una masa crítica de personas calificadas y cultas, ningún país podrá garantizar un auténtico desarrollo endógeno y sostenible, los países en desarrollo y los países pobres, en particular, no podrán acortar la brecha que los separa de los países desarrollados. El intercambio de conocimientos, la cooperación internacional y las nuevas tecnologías pueden brindar nuevas oportunidades de reducir estas disparidades.

Dado el alcance y el ritmo de las transformaciones anteriores, la sociedad cada vez tiende más a fundarse en el conocimiento. Lo anterior le confiere a la educación superior y a la investigación un papel fundamental en el desarrollo cultural, socioeconómico y ecológicamente sostenible de los individuos, las comunidades y las naciones. Por consiguiente, la propia educación superior debe estar en capacidad de contribuir significativamente para que la sociedad contemporánea, que en la actualidad vive una profunda crisis de valores, pueda transcender las consideraciones meramente económicas y asumir compromisos de moralidad, solidaridad y justicia cada vez más arraigados.

\section{La educación superior hacia el futuro}

Las actuales condiciones que experimenta nuestra sociedad obligan a la universidad a revisar el conjunto de relaciones que mantiene con la comunidad nacional de la que forma parte. Cada vez se le presentan a la universidad nuevas demandas y exigencias, pero también estímulos y desafíos, a los cuales debe responder con visión y capacidad. Se impone entonces un proceso de autocrítica y evaluación de sus funciones naturales: Docencia, Investigación y Extensión y de sus responsabilidades con el estado y la sociedad.

El diálogo de la universidad con el estado, debe basarse en el compromiso de la elaboración de un proyecto político nacional y posteriormente en su implementación. Dada la importancia de la investigación científica y tecnológica en el desarrollo económico y social del país, la universidad debe colaborar en la orientación de cualquier proyecto político nacional. Los países económicamente desarrollados han depositado en las universidades, no solo la responsabilidad de la formación de sus profesionales, sino la investigación científica necesaria para alcanzar tal desarrollo. Este hecho le da a la investigación en la universidad un papel fundamental.

En el actual momento histórico no puede concebirse a la universidad como una isla completamente desligada de un proyecto nacional. Esta debe responder 
a la sociedad por la enorme cantidad de recursos que recibe, sobre todo en países subdesarrollados como los de nuestra región. El reto para la universidad será cumplir con este compromiso sin sacrificar su autonomía.

\section{Conclusiones y recomendaciones}

\section{Gestión universitaria}

Se impone considerar los fundamentos de la gestión estratégica. Deben elaborarse planes a corto, mediano y largo plazo que deberán revisarse y adaptarse constantemente en función de la interacción con los diferentes sectores de la sociedad. En esta labor, deben determinarse prioridades, metas y objetivos, teniendo en cuenta fortalezas, debilidades, amenazas y oportunidades.

La autonomía universitaria es una barrera que impide la intromisión en las políticas universitarias de los gobiernos de turno, pero esto no elimina la responsabilidad que tiene la universidad de rendir cuentas claras de su quehacer a la sociedad.

Uno de los problemas más serios que ha tenido la universidad en nuestros países ha sido la excesiva e ineficiente burocracia. Con los medios tecnológicos actuales y los mecanismos administrativos modernos la universidad debe mejorar sustancialmente la calidad de sus servicios.

\section{Financiamiento}

A pesar de la garantía que el estado y la sociedad tienen con las universidades públicas de proporcionarles los recursos que necesiten para su funcionamiento, estamos ante un contexto de reducción de gastos estatales, lo que obliga a la universidad a buscar mecanismos que le permitan obtener más recursos materiales y económicos y disminuir así, por lo menos en parte, su dependencia económica del estado. Es necesario realizar un enorme esfuerzo para la obtención de rentas propias, administradas con la claridad y la eficiencia que una inversión de este tipo requiere. De esta forma, puede afirmarse que el funcionamiento universitario debe basarse en la calidad. Debe exigirse excelencia en el personal docente, en los estudiantes y en los funcionarios administrativos.

Debe establecerse un pago diferenciado por parte de los estudiantes, en función de sus condiciones socioeconómicas. Quien pueda pagar, debe pagar. Estos fondos deberían orientarse a fortalecer los servicios estudiantiles, así como el acceso de estudiantes de menores recursos económicos. 
La admisión a la universidad debe basarse por un lado en el favorecimiento de aquellas carreras que determinen las prioridades nacionales y por otro en la calidad de los estudiantes independientemente de su condición socioeconómica. En este sentido deben establecerse criterios de admisión que tomen en cuenta ambos factores.

Por otro lado, deben hacerse esfuerzos por disminuir el tiempo de graduación de los estudiantes. Estudios recientes demuestran que los estudiantes costarricenses tardan mucho más de cuatro años en obtener su bachillerato universitario. En el caso de las licenciaturas, el promedio de duración es de aproximadamente once años. Jofré (1998, p.266). La permanencia de los estudiantes en la institución debe estar regida por el criterio del mejor rendimiento académico. Pero esta exigencia debe ir acompañada de servicios de apoyo tales como: bibliotecas, atención psicológica, becas cuando sea necesario, servicios de salud, etc.

Se requieren más esfuerzos por mejorar el acceso de las mujeres a la educación superior. Consolidar plenamente su participación en todos los niveles e incrementar su participación activa en los órganos de dirección universitaria.

\section{La docencia en la universidad}

Muchos docentes universitarios no tienen claro porqué enseñan, no tienen claridad sobre los métodos que utilizan, ni del proyecto del ser humano que quieren formar.

Dado el avance de la ciencia, las universidades requieren docentes que orienten a los estudiantes para que ubiquen y usen la información. No se trata de que desaparezca el contenido, sino que tendrá prioridad no tanto la transmisión del mismo, sino cómo conseguir esa información. Los docentes deben entonces clarificar sus métodos de trabajo para que orienten a los estudiantes a ubicar y aprovechar la información. La investigación debe estar vinculada a la docencia, para ello los docentes tienen que ser investigadores constantes de su propia labor.

Para que la docencia cumpla su papel es necesario crear nuevas formas de implementarla. Debe emplearse la duda en lugar de la transmisión de viejos conocimientos. El profesor debe estimular en sus estudiantes el cuestionamiento, la búsqueda de respuestas, la investigación, la capacidad de análisis, la formulación de críticas y explicaciones propias. Estas nuevas estrategias didácticas deben planearse con gran seriedad y responsabilidad pues de lo contrario pueden provocar en el estudiante reacciones de rechazo dada la inseguridad que sienten 
ante el cambio y al asumir mayores cuotas de responsabilidad en su propio aprendizaje.(Buarque, 1991, p.107)

Por otro lado deben efectuarse procesos de selección del personal docente basados exclusivamente en el rigor y la calidad académicas, eliminando cualquier influjo político. Además, el ascenso en la carrera académica debe hacerse a través de un estatuto de exigencia en el trabajo y de estímulo a la producción de calidad, tanto en la docencia como en la investigación. Lo anterior debe complementarse con una recuperación del status del profesor universitario, aquí se incluyen aspectos como los salarios y los estímulos laborales que deben ser significativos de acuerdo a la productividad académica.

\section{La investigación universitaria}

La Educación Superior debe ser el lugar donde se realice prioritariamente la investigación de alta calidad. Esta tarea debe recibir un gran apoyo institucional y un creciente presupuesto y debe estar ligada a la solución de los problemas nacionales.

En nuestros países la investigación realizada en el sistema universitario ha tenido poco impacto en la sociedad, quizás debido a su poca eficacia dada la gran separación de la universidad con los sectores activos de la economía. Debe exigirse a la universidad que la investigación produzca conocimiento relevante, que sus resultados sean adecuadamente divulgados y que tenga impacto en los sectores que podrían beneficiarse con el conocimiento producido. (Jofré,1998, p.229)

Las universidades deben procurar una mayor integración de los grupos dedicados a la investigación. Muchas veces prevalecen actitudes gremialistas y sectarias que deben ser superadas por un sentido más colectivo de trabajo. La ciencia hoy día es una labor de equipo en la que se deben sumar las fortalezas de los diversos investigadores.

Las universidades deben fortalecer sus sistemas de posgrados, de modo que se garantice la formación de recursos humanos de alto nivel en ciencia y tecnología. El posgrado debe convertirse en un eje fundamental del desarrollo universitario, asimismo debe superar los estrechos esquemas gremialistas para convertirse en un ejemplo de integración académica y de trabajo multidisciplinario.

\section{Vinculación con el sector externo}

La universidad está en la obligación de contribuir a la superación cultural de la sociedad, para esto debe asumir tareas de divulgación del conocimiento, 
proyección literaria, artística, deportiva. ecológica, etc. Los modernos medios de comunicación proporcionan mecanismos idóneos para realizar estas tareas.

La universidad debe crear los mecanismos para orientar y dirigir las reformas educativas que requieren nuestros países. Debe mejorar la formación del personal docente y realizar investigación sobre educación. Para ello debe aportar los recursos humanos profesionales y los conocimientos teóricos que se requieren para cumplir con esta misión.

La educación superior debe reforzar sus funciones de servicio a la sociedad, en particular las encaminadas a erradicar la pobreza, la intolerancia, la violencia, el analfabetismo, el hambre, el deterioro del medio ambiente y las enfermedades.

Deben buscarse los mecanismos que posibiliten de una manera expedita y ágil la transferencia del conocimiento a los diferentes sectores de la sociedad que lo requieren. Las necesidades en estos casos deben partir fundamentalmente de la sociedad.

\section{Referencias}

Bailey,T. (1994). Educación, Cambio Tecnológico y Crecimiento Económico. Revista La Educación. (119). 461-475.

Buarque, C (1991). La Universidad en la Frontera del Futuro. San José, Costa Rica: EUNA.

Centro de Estudios para el Perfeccionamiento de la Educación Superior (1995). La Universidad Latinoamericana en el fin de siglo.México: Colección UDUAL.

Jofré, A (1998). La Universidad en América Latina. Cartago, C.R: Editorial Tecnológica de Costa Rica.

Licha, I (1996). La Investigación y las Universidades Latinoamericanas en el Umbral del Siglo XXI: Los Desafíos de la Globalización. México: Colección UDUAL.

Proyecto Estado de la Nación (1999). Estado de la Nación en Desarrollo Humano sostenible. San José, C.R: Editorama.

Seminario Universidad y Desarrrollo (1995). Univesidad y Desarrollo: a contrapelo de los tiempos. San José, C.R: Oficina de Publicaciones de la Universidad de Costa Rica. 
Tunnermann, C (1998). Transformación de la Educación Superior: Retos y Perspectivas Heredia, C.R: EUNA

Tunnermann, C (1997). La Educación Superior frente al Cambio. San José, C.R: EDUCA/CSUCA. 